\title{
Diagnostic Accuracy of Fine Needle Biopsy for Metastatic Melanoma and Its Implications for Patient Management
}

\author{
Anna Doubrovsky, ${ }^{1}$ Richard A. Scolyer, ${ }^{1,2,5,6}$ Rajmohan Murali, ${ }^{1,2,5,6}$ Paul R. McKenzie, ${ }^{2}$ \\ Geoffrey F. Watson, ${ }^{2}$ C. Soon Lee, ${ }^{2,5}$ Duncan J. McLeod, ${ }^{2}$ William H. McCarthy, ${ }^{1,4,6}$ \\ Roger F. Uren, ${ }^{3}$ Jonathan R. Stretch, ${ }^{1,4,6}$ Robyn P. Saw, ${ }^{1,4,6}$ and John F. Thompson ${ }^{1,4,6}$ \\ ${ }^{1}$ Sydney Melanoma Unit, Sydney Cancer Centre, Royal Prince Alfred Hospital, Camperdown, NSW 2050, Australia \\ ${ }^{2}$ Department of Anatomical Pathology, Royal Prince Alfred Hospital, Camperdown, NSW, Australia \\ ${ }^{3}$ Nuclear Medicine and Diagnostic Ultrasound and Discipline of Medicine, The University of Sydney, Sydney, NSW, Australia \\ ${ }^{4}$ Discipline of Surgery, Faculty of Medicine, The University of Sydney, Sydney, NSW, Australia \\ ${ }^{5}$ Discipline of Pathology, Faculty of Medicine, The University of Sydney, Sydney, NSW, Australia \\ ${ }^{6}$ Melanoma and Skin Cancer Research Institute, Royal Prince Alfred Hospital, New South Wales, Camperdown, Australia
}

\begin{abstract}
Background: The use of fine needle biopsy (FNB) for the diagnosis of metastatic melanoma can lead to the early removal and treatment of metastases, reduce the frequency of unnecessary surgery, and facilitate the staging of patients enrolled in clinical trials of adjuvant therapies. In this study, the accuracy of FNB for the diagnosis of metastatic melanoma was investigated.

Methods: A retrospective cohort study was performed with 2204 consecutive FNBs performed on 1416 patients known or suspected to have metastatic melanoma. Almost threequarters (1582) of these FNBs were verified by either histopathologic diagnosis following surgical resection or clinical follow-up.

Results: FNB for metastatic melanoma was found to have an overall sensitivity of $92.1 \%$ and a specificity of $99.2 \%$, with 69 false-negative and 5 false-positive findings identified. The sensitivity of the procedure was found to be influenced by six factors. The use of immunostains, reporting of the specimen by a cytopathologist who had reported $>500$ cases, lesions located in the skin and subcutis, and patients with ulcerated primary melanomas were factors associated with a significant improvement in the sensitivity of the test. However, FNBs performed in masses located in lymph nodes of the axilla and FNBs that required more than one needle pass to obtain a sample were far more likely to result in false-negative results.
\end{abstract}

Conclusions: FNB is a rapid, accurate, and clinically useful technique for the assessment of disease status in patients with suspected metastatic melanoma.

Key Words: Cytology—Diagnosis—Diagnostic accuracy_Fine needle biopsy-MelanomaPathology.

Fine needle biopsy (FNB) is frequently used in the diagnostic workup of clinically or radiologically

Received August 22, 2006; accepted November 15, 2006; published online: November 8, 2007.

Address correspondence and reprint requests to: John F. Thompson; Sydney Melanoma Unit, Sydney Cancer Centre, Royal Prince Alfred Hospital, Camperdown, NSW 2050, Australia; E-mail: john.thompson@smu.org.au

Published by Springer Science+Business Media, LLC @ 2007 The Society of Surgical Oncology, Inc. detected mass lesions that are suspicious for metastatic melanoma. By determining whether they represent metastatic melanoma, the use of FNB in melanoma patients can expedite detection of metastases, leading to earlier removal and treatment; facilitate the staging of patients enrolled in clinical trials of adjuvant therapies (particularly in deep-seated lesions); reduce the frequency of unnecessary surgery; and assist in the planning of the most appropriate surgery. 
FNB is a swift, minimally invasive and cost-effective technique employed in the diagnostic workup of mass lesions occurring in a wide variety of organs. ${ }^{1,2}$ The technique has been extensively evaluated in assessing the nature of lesions located in the tissues of the breast, thyroid, lung, liver, pancreas, lymph nodes, salivary glands, and kidneys, among other locations. $^{3-6}$

There have been several previous case studies assessing the use of FNB in patients with melanoma. The majority of these studies lacked sufficient case numbers to precisely determine the diagnostic accuracy of FNB. ${ }^{7-10}$ However, in 1986, Perry and colleagues ${ }^{11}$ analyzed almost 300 FNBs from melanoma patients and found the procedure to be accurate, with a sensitivity of $86.5 \%$ and a specificity of $96.1 \%$. Since this study, there have been important changes in the procedure, including improvements in immunochemical characterization and radiological guidance. In 2000, Voit and colleagues ${ }^{12}$ published a study of 739 FNBs from melanoma patients with palpable suspicious lymph nodes or small lesions that were only detectable by ultrasound B-scan examination. Although the authors reported a sensitivity of $97.8 \%$ and a specificity of $100.0 \%$, the sensitivity depended on lesion size.

The objective of this retrospective cohort study was to evaluate the diagnostic accuracy of the FNB procedure in the detection of metastatic melanoma. To accomplish this, a very large consecutive sample was collected of FNBs performed on melanoma patients who attended the Sydney Melanoma Unit (SMU), Sydney, Australia. This large sample also allowed evaluation of the effect of several clinicopathologic features and factors related to the procedure on the diagnostic accuracy of FNB.

\section{MATERIALS AND METHODS}

\section{Patients}

For all patients with melanoma who attended the SMU and gave informed consent, clinical and histologic details of their disease were recorded on the SMU database, and follow-up information was entered prospectively. Patients with suspicious clinically palpable or radiologically identified mass lesions, detected by a variety of imaging modalities, were further investigated by FNB. All SMU patients who underwent an FNB that had been reported by cytopathologists from the Department of Anatomical Pathology at the Royal Prince Alfred Hospital in
TABLE 1. Patient characteristics

\begin{tabular}{lrr}
\hline Characteristic & $\mathrm{n}$ & $\%$ \\
\hline Sex & & \\
Male & 888 & 62.7 \\
Female & 528 & 37.3 \\
Age at melanoma diagnosis (y) & & \\
10-30 & 110 & 7.8 \\
$31-40$ & 135 & 9.5 \\
$41-50$ & 256 & 18.1 \\
$51-60$ & 303 & 21.4 \\
$61-70$ & 302 & 21.3 \\
$70-80$ & 219 & 15.5 \\
$81+$ & 62 & 4.4 \\
Unknown & 29 & 2.0 \\
Cancer diagnosis & & \\
Other cancer diagnoses & 159 & 11.2 \\
More than one other cancer diagnoses & 15 & 1.1 \\
No. of primary melanoma lesions & & \\
1 & 1236 & 87.3 \\
2 & 148 & 10.4 \\
$\geq 3$ & 32 & 2.3 \\
No. of FNBs & & \\
1 & 953 & 67.3 \\
2 & 287 & 20.3 \\
3 & 98 & 6.9 \\
>3 & 78 & 5.5 \\
Total no. of patients & 1416 & 100.0 \\
\hline
\end{tabular}

FNB, fine needle aspiration biopsy.

Sydney, Australia, between January 1992 and December 2002 were identified from the SMU database, and their clinical records and FNB reports were reviewed. Details of the patients are provided in Table 1.

\section{FNB Procedure}

For palpable lesions, the FNBs were performed by the reporting cytopathologist or a trainee pathologist under their supervision. Following localization and stabilization of the lesion with one hand, a hollow bore needle $(22 \mathrm{G}, 23 \mathrm{G}$, or $25 \mathrm{G})$ was inserted directly into the mass and the needle was moved swiftly in and out for approximately 10 seconds. Aspiration with a syringe was not used in the vast majority of cases, hence our preference for the term 'fine needle biopsy' and 'fine needle aspiration biopsy'. In our experience, the use of a needle without an attached syringe allows better control of the movement of the needle. Furthermore, aspiration often yields blood, promotes clotting and hampers optimal interpretation of cytologic detail. The procured material was ejected from the needle onto glass slides by pushing air from a syringe through the needle. The material was spread evenly across the slide using another glass slide. One slide was air-dried and stained immediately with Diff-Quik (Lab Aids, Narrabeen, NSW, Aus- 
TABLE 2. Categories of cytodiagnoses

\begin{tabular}{|c|c|c|c|c|}
\hline Characteristic & Total $n$ & $\%$ & Confirmed & Not confirmed \\
\hline \multicolumn{5}{|l|}{ Positive for Metastatic Melanoma } \\
\hline Malignant cells-melanoma & 1089 & 49.4 & 805 & 284 \\
\hline \multicolumn{5}{|l|}{ Suspicious for Metastatic Melanoma } \\
\hline Malignant cells - suspicious for melanoma & 44 & 2.0 & 39 & 5 \\
\hline Malignant cells - unknown cancer & 33 & 1.5 & 22 & 11 \\
\hline Suspicious for malignancy & 40 & 1.8 & 35 & 5 \\
\hline \multicolumn{5}{|l|}{ Negative for Metastatic Melanoma } \\
\hline Malignant cells - other cancer & 92 & 4.2 & 47 & 45 \\
\hline No malignant cells - other cells present & 416 & 18.9 & 308 & 108 \\
\hline No malignant cells - scant other cells & 296 & 13.4 & 204 & 92 \\
\hline No malignant cells - no other cells & 175 & 7.9 & 122 & 53 \\
\hline No malignant cells - procedure not performed & 19 & 0.9 & 0 & 19 \\
\hline Total & 2204 & 100 & 1582 & 622 \\
\hline
\end{tabular}

tralia) and another fixed in alcohol and later stained by the Papanicolaou method. ${ }^{2}$ Residual material was washed into Hanks balanced salt solution for later preparation of cell blocks using the serum-prothrombin method ${ }^{2}$ or by cytocentrifuge preparations, to be used for immunochemistry. The air-dried slides were examined by the cytopathologist at the time of the procedure. Further passes were performed if necessary, depending on the amount and type of cellular material obtained.

\section{Review of Clinical Material and Follow-up}

The accuracy of the FNB procedure in diagnosing metastatic melanoma was evaluated by two reference standards: a) histopathologic evaluation of the excised lesion (1120 cases) or b) follow-up in those cases for which histologic material was not available (462 cases). The duration of follow-up was 6 months or greater (mean 50.2 months, median 45.7 months, range 6.1-144.4 months) in 456 cases. In six cases, the length of follow-up was less than 6 months (mean 4.2 months, median 4.0 months, range 3.0-5.7 months). The mass was considered benign if it was stable in size or resolved after clinical follow-up.

\section{Cytodiagnosis}

Cytodiagnoses were categorized as positive, suspicious, or negative for metastatic melanoma (Table 2). Cases were considered positive for metastatic melanoma if the specimen included sufficient numbers of well-preserved malignant cells with typical cytological features, pigment, and/or confirmatory immunochemistry for a confident diagnosis of melanoma to be made. On verification, these samples were classified as truly positive $(\mathrm{n}=800,50.5 \%)$ or falsely positive $(\mathrm{n}=5,0.3 \%)$.
Samples that contained cells from unclassified/ unspecified malignancies or cases categorized as suspicious for melanoma (those with small numbers of atypical cells, poorly preserved cells, and cells that lacked specific features of melanoma, such as cytoplasmic pigment, and where insufficient material was present for immunochemistry) were classified as suspicious for metastatic melanoma. These were determined to be true suspicious ( $\mathrm{n}=78,4.9 \%)$ or falsely suspicious $(\mathrm{n}=18,1.1 \%)$ results after verification.

FNBs classified as negative for metastatic melanoma contained no material that could be diagnosed as metastatic melanoma. They included cases containing malignant cells diagnosed as another neoplasm or b) various amounts of cellular material from the tissue of the site that was sampled. After verification, these procedures were found to be either true negative $(\mathrm{n}=612,38.7 \%)$ or false negative $(\mathrm{n}=69$, $4.4 \%)$.

\section{Statistical Analysis}

All analyses were performed using the S-PLUS software package (Insightful Corporation, Seattle, WA) and Microsoft Excel, version 2000 (Microsoft, Redmond, WA). Diagnostic accuracy of FNB for metastatic melanoma was measured by the sensitivity and specificity of the test.

The effect of the type of tissue involved by melanoma, anatomic location, and 15 clinicopathologic and procedural factors were analyzed. These factors included features of the primary melanoma such as tumor thickness, dermal mitotic rate, presence of ulceration, predominant cell type and histopathologic subtype of melanoma; patient attributes such as sex, American Joint Committee on Cancer (AJCC)/ International Union Against Cancer (UICC) stage, ${ }^{13}$ age at FNB and location of the FNB (local or distant to the primary lesion); aspects of the FNB procedure 
TABLE 3. Fine needle biopsy procedures that could not be performed

\begin{tabular}{|c|c|c|c|}
\hline Location & Explanation for failure & Confirmation & Histology \\
\hline Left neck & Unable to locate lesion & Negative-follow-up & - \\
\hline Left face (subcutis) & Pain & Not confirmed & - \\
\hline Right face & Unable to locate lesion & Not confirmed & - \\
\hline Left neck (LN) & Unable to locate lesion & Negative-surgery & No evidence of malignancy \\
\hline Right breast & Adjacent to prothesis & Negative-follow-up & - \\
\hline Right neck & Pain & Not confirmed & - \\
\hline Right axilla (LN) & Unable to locate lesion & Negative-follow-up & - \\
\hline Right neck & Pain & Negative - surgery & No evidence of malignancy \\
\hline Left neck (LN) & Pain & Negative - follow-up & - \\
\hline Left neck (LN) & Unable to locate lesion & Negative-follow-up & - \\
\hline Right groin & Pain & Negative - surgery & No evidence of malignancy \\
\hline Left axilla (LN) & Unable to locate lesion & Positive - surgery & Melanoma \\
\hline Right axilla (LN) & Unable to locate lesion & Negative-follow-up & - \\
\hline Right neck (LN) & Pain & Not confirmed & - \\
\hline Left axilla (LN) & Unable to locate lesion & Not confirmed & - \\
\hline Left neck & Unable to locate lesion & Negative-follow-up & - \\
\hline Thyroid & Unable to locate lesion & Negative-follow-up & - \\
\hline Right axilla (LN) & Unable to locate lesion & Negative-follow-up & - \\
\hline Right sternum (LN) & Unable to locate lesion & Not confirmed & - \\
\hline
\end{tabular}

LN, lymph node.

such as number of needle passes, needle size, the experience (based on caseload) of the reporting cytopathologist; year of procedure, use of immunostains; and presence of necrosis. Statistical significance was determined by the $95 \%$ confidence intervals (95\% CIs) of these parameters. When comparing different samples, a two-sample test for binomial proportions was used. All equations used have been described elsewhere. ${ }^{14,15}$

\section{RESULTS}

In the 11-year study period, 2204 consecutive FNBs were performed in 1416 patients. Nineteen FNBs $(0.9 \%$ of all FNB cases) were excluded from the analysis because the procedure could not be performed, i.e., no aspirate was obtained (Table 3). In half of these cases, the lesion could not be located (inappropriate referrals), but patient pain tolerance and difficulties with the procedure also contributed.

Verification of the remaining 2185 FNBs was partial; in 1582 (71.8\%), the true disease status was confirmable by either histopathologic evaluation of the excised lesion or by clinical follow-up (Fig. 1). A total of $1120 \mathrm{FNBs}$ were verified by histopathologic evaluation of the excised lesion; all confirmed positive FNBs and FNBs with false-positive or negative results were verified histologically. In those cases (mostly FNBs with negative results) where histopathologic material was not available (462 cases), verification was made by clinical follow-up. The mass was considered benign if it was stable in size or if it resolved after clinical follow-up. The duration of follow-up was $>6$ months in 456 cases and 3 to 6 months in 6 cases. Of the verified FNBs, 1435 (90.7\%) were palpation-guided FNBs performed by trainee cytopathologists or cytopathologists, while 147 (9.3\%) were image-guided FNBs performed by radiologists or other physicians.

A total of 603 FNBs (27.4\%) could not be verified; this was due to loss to follow-up, particularly by death in patients with advanced metastatic melanoma, uncertainty regarding sites (where FNBs from more than one site were obtained at the same visit to the clinic), and where there were multiple metastases, which led to uncertainty in lesion location and the correlation of FNB and histopathologic results. Unconfirmed FNBs differed from confirmed procedures in several ways. They were more likely to be from lesions located in visceral organs or to be in patients with AJCC/UICC stage IV disease. FNBs with inconclusive cytodiagnoses were more likely to be followed up with clinical observation or further biopsy.

Confirmation of FNBs was unaffected by the year of procedure, the number of needle passes during sampling, the use of immunostains, the reporting cytopathologist, or the age or sex of the patient.

Approximately $12 \%$ of the patients were diagnosed with additional cancers (most commonly breast cancer, colorectal cancer, and chronic lymphocytic leukaemia), with some suffering from multiple types of other cancers. Multiple primary melanomas occurred 


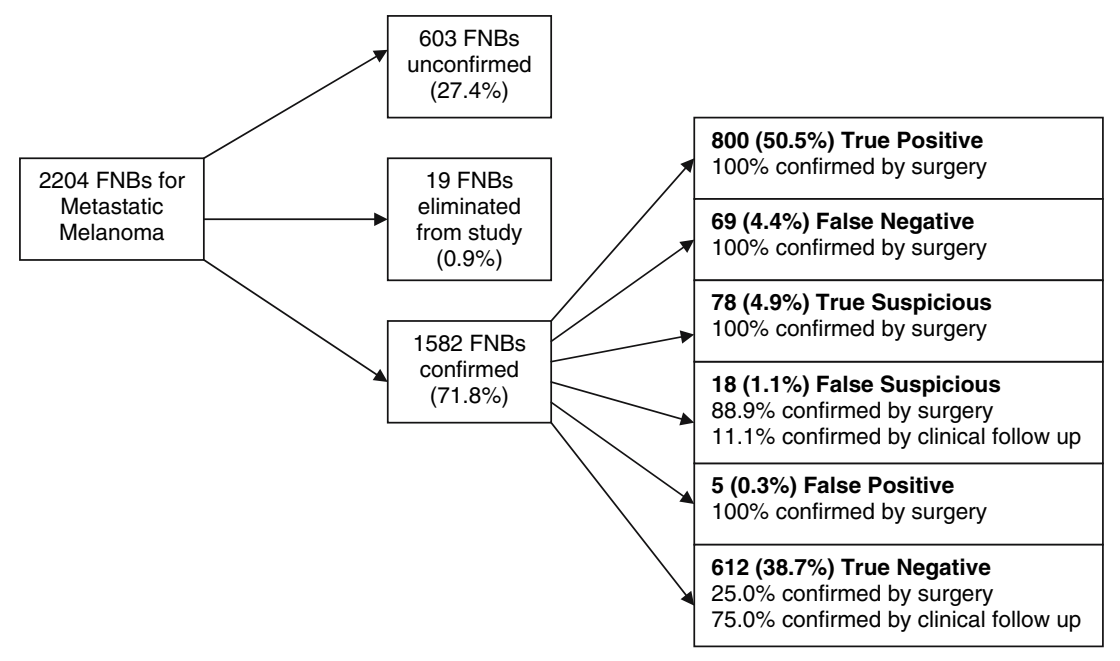

FIG. 1. Flow diagram showing fine needle biopsy (FNB) result distribution.

TABLE 4. False-positive fine needle biopsy findings for metastatic melanoma

\begin{tabular}{llll}
\hline Cytodiagnosis & Histology & Location & Comment \\
\hline Melanoma & Metastatic adenocarcinoma & Right axillary (LN) & - \\
Melanoma & Metastatic papillary carcinoma & Left supraclavicular fossa (LN) & - \\
Melanoma & Hematoma & Left axilla & - \\
Melanoma & Chronic osteomyelitis & Left skull (bone) & S100 positive ${ }^{a}$ \\
Melanoma & Metastatic adenocarcinoma & Right axilla & - \\
\hline
\end{tabular}

LN, lymph node.

${ }^{a}$ S100-positive histiocytes were identified in the excision specimen, which probably caused the misdiagnosis.

in an eighth of this patient population. Almost a third of the patients underwent multiple FNBs. These procedures were performed both concurrently and sequentially.

There were 1582 FNB procedures for metastatic melanoma with histologic verification or clinical follow-up. The overall sensitivity was $92.1 \%$ (95\% CI, 93.7-90.0) and the specificity was $99.2 \%$ (95\% CI, 99.7-98.1). Five cases were determined to be false positive, resulting in a false-positive rate of $0.6 \%$ (Table 4). The false-negative rate was $10.2 \%$, with no metastatic melanoma identified in 69 FNB cases in which metastatic melanoma was identified by later histologic evaluation (Fig. 2).

The large numbers of confirmed cases permitted detailed analysis of this procedure for metastatic melanoma. The effect of FNB site was studied (Table 5). Lymph node tissue was the most common site for FNB evaluation, with 926 procedures, 753 of them confirmed. The sensitivity and specificity of FNB for metastatic melanoma in lymph nodes were not significantly different compared with that for all sites. However, the sensitivity for FNBs performed on lymph nodes located in the axilla was approximately $9 \%$ less compared with FNBs performed on

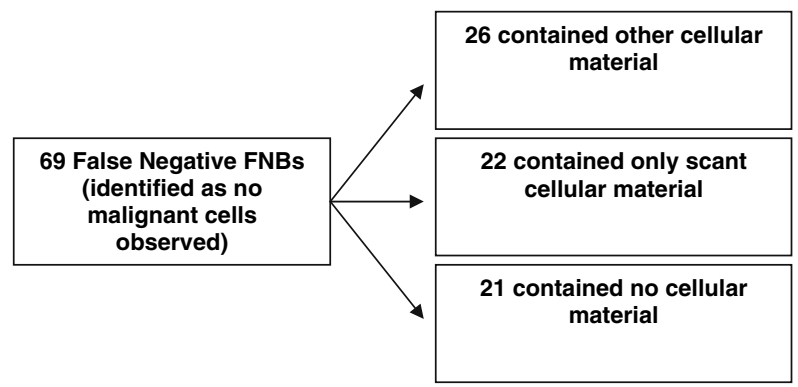

FIG. 2. Distribution of false-negative fine needle biopsy (FNB) cytodiagnoses.

lymph nodes in other locations $(\mathrm{z}=-3.9, \mathrm{P}=$ 0.0001 ). Increased FNB sensitivity (by $4 \%$ ) was found when the procedure was performed on lesions located in the skin and subcutis compared with other sites. However, this was only of borderline significance $(\mathrm{z}=1.9, \mathrm{P}=0.05)$. Too few confirmed FNBs were conducted on visceral organs $(\mathrm{n}=79)$ to allow conclusions to be drawn regarding the success of the procedure in these locations.

Fifteen clinicopathologic and procedural factors were analyzed to assess their effect on the diagnostic accuracy of FNB for metastatic melanoma (Table 6). 
TABLE 5. Diagnostic accuracy of FNB: Effect of tissue type and anatomic location

\begin{tabular}{|c|c|c|c|c|c|c|c|c|c|c|c|c|c|}
\hline Location & $\mathrm{n}$ & Confirmed & $\%$ & $\mathrm{TP}$ & $\mathrm{FN}$ & TS & FS & FP & $\mathrm{TN}$ & $\mathrm{Sn}$ & $(95 \% \mathrm{CI})$ & $\mathrm{Sp}$ & $(95 \% \mathrm{CI})$ \\
\hline All FNB & 2204 & 1582 & 71.8 & 800 & 69 & 78 & 18 & 5 & 612 & 0.92 & $(0.90-0.94)$ & 0.99 & $(0.98-1.00)$ \\
\hline Lymph nodes & 926 & 753 & 81.3 & 413 & 43 & 41 & 7 & 3 & 246 & 0.91 & $(0.88-0.93)$ & 0.99 & $(0.97-1.00)$ \\
\hline Neck & 235 & 185 & 78.7 & 104 & 3 & 15 & 4 & 1 & 58 & 0.97 & $(0.92-0.99)$ & 0.98 & $(0.91-1.00)$ \\
\hline Axilla & 383 & 313 & 81.7 & 155 & 29 & 12 & 2 & 2 & 113 & $0.84^{a}$ & $(0.78-0.89)$ & 0.98 & $(0.94-1.00)$ \\
\hline Groin & 274 & 233 & 85.0 & 144 & 11 & 11 & 0 & 0 & 67 & 0.93 & $(0.88-0.96)$ & 1.00 & - \\
\hline Other & 34 & 22 & 64.7 & 10 & 0 & 3 & 1 & 0 & 8 & 1.00 & - & 1.00 & - \\
\hline Skin and subcutis & 711 & 504 & 70.9 & 270 & 17 & 26 & 4 & 0 & 187 & $0.94^{b}$ & $(0.91-0.96)$ & 1.00 & - \\
\hline Head and neck & 131 & 98 & 74.8 & 54 & 5 & 7 & 0 & 0 & 32 & 0.92 & $(0.82-0.96)$ & 1.00 & - \\
\hline Trunk & 288 & 196 & 68.1 & 101 & 4 & 6 & 2 & 0 & 83 & 0.96 & $(0.91-0.99)$ & 1.00 & - \\
\hline Limbs & 292 & 210 & 71.9 & 115 & 8 & 13 & 2 & 0 & 72 & 0.93 & $(0.88-0.97)$ & 1.00 & - \\
\hline Visceral organs & 176 & 79 & 44.9 & 30 & 3 & 5 & 2 & 0 & 39 & 0.91 & $(0.76-0.97)$ & 1.00 & - \\
\hline Liver & 56 & 22 & 39.3 & 7 & 1 & 1 & 0 & 0 & 13 & 0.88 & $(0.53-0.98)$ & 1.00 & - \\
\hline Lung & 94 & 43 & 45.7 & 18 & 2 & 4 & 1 & 0 & 18 & 0.90 & $(0.70-0.97)$ & 1.00 & - \\
\hline Other & 391 & 246 & 62.9 & 87 & 6 & 6 & 5 & 2 & 140 & 0.94 & $(0.87-0.97)$ & 0.99 & $(0.95-1.00)$ \\
\hline
\end{tabular}

TP, true positive; FN, false negative; TS, true suspicious; FN, false suspicious; FP, false positive; TN, true negative; SN, sensitivity; SP, specificity; 95\% CI, 95\% confidence interval.

${ }^{a}$ Axilla lymph nodes had significantly reduced sensitivity compared with other sites $(\mathrm{z}=-3.9, \mathrm{P}=.0001)$.

${ }^{b}$ Skin and subcutis FNBs had significantly increased sensitivity compared with other sites $(\mathrm{z}=1.9, \mathrm{P}=.05)$.

The FNB confirmation rate was similar for all clinicopathologic factors, with the exception of $\mathrm{AJCC} /$ UICC stage and location (distant or regional to the primary lesion), as described above.

Patients with an ulcerated primary lesion experienced slightly better FNB test sensitivity compared to those with no ulceration $(\mathrm{z}=1.9, \mathrm{P}=0.05)$. AJCC/ UICC stage, anatomic location, year of procedure, needle size, sex, age at FNB, primary lesion thickness, dermal mitotic rate, predominant cell type, histologic subtype of melanoma, and presence of necrosis did not affect the diagnostic accuracy of the test (Table 6).

Three factors related to the conduct of the procedure affected FNB test accuracy. These were the number of needle passes needed to collect the sample, the number of FNBs for metastatic melanoma the cytopathologist had reported during the study period (caseload), and the use of immunostains. Samples that required only one attempt at FNB had a sensitivity increase by $>10 \%$ compared to samples with more than one attempt $(\mathrm{z}=4.8, \mathrm{P}<0.001)$. Sensitivity fell in a linear manner with each subsequent attempt (Fig. 3). Cytopathologists who had caseloads of $>500$ FNBs ( 2 cytopathologists) had greater FNB test sensitivity by about $5 \%$ compared to those who had reported $<500$ FNBs (16 cytopathologists) $(z=$ $2.4, \mathrm{P}=0.02)$. Finally, FNB tests in which immunostains were used had better sensitivity $(\mathrm{z}=4.8, \mathrm{P}$ $<0.001$ ) (Table 6).

\section{DISCUSSION}

Metastatic melanoma is an aggressive tumor with a high mortality rate. Patients with primary melanomas that are at high risk of metastasizing who attend the SMU are followed closely for evidence of metastatic disease with radiological investigations and regular clinical follow-up. Early surgery for metastatic disease may lead to longer disease-free periods and may ultimately improve survival in some of these patients. This underscores the importance of early detection of metastatic disease.

In some melanoma treatment centers, FNB has been used for many years to verify clinically suspicious lesions and radiological abnormalities in patients with melanoma, prior to traumatic or costly surgical or adjuvant treatment. FNB is a cost-effective, rapid procedure that is well tolerated by patients when performed by proficient operators. ${ }^{2}$ However, the ability of the test to accurately diagnose suspicious lesions as metastatic melanoma has been infrequently studied.

Sensitivity and specificity are measures that are used extensively in the FNB literature. They were chosen as the best measures of diagnostic accuracy of FNBs in patients with melanoma because of the binary quality of the data. ${ }^{16}$ In this study, a large number of consecutive FNB procedures (2204) for metastatic melanoma were analyzed, with 1582 procedures confirmed by either histopathology after surgical resection or by clinical follow-up. This cohort represents more FNB procedures in melanoma patients than all previous reported series combined. ${ }^{7-12}$ The overall sensitivity of FNB for metastatic melanoma was $92.1 \%$, and the specificity was $99.2 \%$. These results are superior to those obtained by Perry and colleagues ${ }^{11}$ in their study of 298 (261 confirmed) cases of metastatic melanoma FNB more than 20 years ago (sensitivity of $86.5 \%$ and specificity 
TABLE 6. Diagnostic accuracy of fine needle biopsy effect of clinicopathologic factors

\begin{tabular}{|c|c|c|c|c|c|c|c|c|c|c|c|c|c|}
\hline Factor & $\mathrm{N}$ & Confirmed & $\%$ & TP & FN & TS & FS & FP & $\mathrm{TN}$ & SN & $(95 \% \mathrm{CI})$ & SP & $(95 \% \mathrm{CI})$ \\
\hline All FNB & 2204 & 1582 & 71.8 & 800 & 69 & 78 & 18 & 5 & 612 & 0.92 & $(0.90-0.94)$ & 0.99 & $(0.98-1.00)$ \\
\hline \multicolumn{14}{|c|}{ AJCC/UICC stage at FNB } \\
\hline Stage I & 400 & 323 & 80.8 & 128 & 9 & 22 & 6 & 3 & 155 & 0.93 & $(0.88-0.97)$ & 0.98 & $(0.95-0.99)$ \\
\hline Stage II & 705 & 569 & 80.7 & 283 & 31 & 24 & 6 & 1 & 224 & 0.90 & $(0.86-0.93)$ & 1.00 & $(0.98-1.00)$ \\
\hline Stage III & 836 & 561 & 67.1 & 310 & 24 & 27 & 5 & 1 & 194 & 0.93 & $(0.90-0.95)$ & 0.99 & $(0.97-1.00)$ \\
\hline Stage IV & 263 & 129 & 49.0 & 79 & 5 & 5 & 1 & 0 & 39 & 0.94 & $(0.87-0.97)$ & 1.00 & $(0.91-1.00)$ \\
\hline \multicolumn{14}{|l|}{ Location } \\
\hline Regional & 1340 & 1077 & 80.4 & 617 & 58 & 58 & 6 & 4 & 334 & 0.91 & $(0.89-0.93)$ & 0.99 & $(0.97-1.00)$ \\
\hline Distant & 864 & 505 & 58.4 & 183 & 11 & 20 & 12 & 1 & 278 & 0.94 & $(0.90-0.97)$ & 1.00 & $(0.98-1.00)$ \\
\hline \multicolumn{14}{|c|}{ Use of immunochemistry } \\
\hline Yes & $583^{\circ}$ & 406 & 69.6 & 316 & 6 & 26 & 8 & 1 & 49 & $0.98^{a}$ & $(0.96-0.99)$ & 0.98 & $(0.90-1.00)$ \\
\hline No & 1621 & 1176 & 72.5 & 484 & 63 & 52 & 10 & 4 & 563 & 0.88 & $(0.86-0.91)$ & 0.99 & $(0.98-1.00)$ \\
\hline \multicolumn{14}{|l|}{ Year } \\
\hline 1992-1994 & 392 & 285 & 72.7 & 144 & 15 & 15 & 5 & 1 & 105 & 0.91 & $(0.85-0.94)$ & 0.99 & $(0.95-1.00)$ \\
\hline 1995-1997 & 555 & 414 & 74.6 & 216 & 15 & 13 & 6 & 1 & 163 & 0.94 & $(0.90-0.96)$ & 0.99 & $(0.97-1.00)$ \\
\hline 1998-2000 & 693 & 500 & 72.2 & 241 & 21 & 26 & 3 & 2 & 207 & 0.92 & $(0.88-0.95)$ & 0.99 & $(0.97-1.00)$ \\
\hline 2001-2002 & 564 & 383 & 67.9 & 199 & 18 & 24 & 4 & 1 & 137 & 0.92 & $(0.87-0.95)$ & 0.99 & $(0.96-1.00)$ \\
\hline \multicolumn{14}{|l|}{ Sex } \\
\hline Male & 1370 & 994 & 72.6 & 500 & 48 & 45 & 10 & 1 & 390 & 0.91 & $(0.89-0.93)$ & 1.00 & $(0.99-1.00)$ \\
\hline Female & 834 & 588 & 70.5 & 300 & 21 & 33 & 8 & 4 & 222 & 0.93 & $(0.90-0.96)$ & 0.98 & $(0.96-0.99)$ \\
\hline \multicolumn{14}{|l|}{ Age at FNB } \\
\hline$\leq 50 \mathrm{y}$ & 572 & 414 & 72.4 & 198 & 12 & 22 & 1 & 2 & 179 & 0.94 & $(0.90-0.97)$ & 0.99 & $(0.96-1.00)$ \\
\hline$>50 \mathrm{y}$ & 1632 & 1168 & 71.6 & 602 & 57 & 56 & 17 & 3 & 433 & 0.91 & $(0.89-0.93)$ & 0.99 & $(0.98-1.00)$ \\
\hline \multicolumn{14}{|c|}{ No. of FNB attempts } \\
\hline 1 & 426 & 309 & 72.5 & 232 & 4 & 3 & 2 & 0 & 68 & $0.98^{b}$ & $(0.96-0.99)$ & 1.00 & $(0.95-1.00)$ \\
\hline 2 & 518 & 371 & 71.6 & 185 & 13 & 21 & 3 & 0 & 149 & 0.93 & $(0.89-0.96)$ & 1.00 & $(0.97-1.00)$ \\
\hline 3 & 315 & 219 & 69.5 & 79 & 13 & 12 & 6 & 0 & 109 & 0.86 & $(0.77-0.92)$ & 1.00 & $(0.97-1.00)$ \\
\hline$\geq 4$ & 192 & 136 & 70.8 & 33 & 17 & 21 & 1 & 1 & 63 & 0.66 & $(0.52-0.78)$ & 0.98 & $(0.92-1.00)$ \\
\hline Unknown & 753 & 547 & 72.6 & 271 & 22 & 21 & 6 & 4 & 223 & 0.92 & $(0.89-0.95)$ & 0.98 & $(0.96-0.99)$ \\
\hline \multicolumn{14}{|l|}{ Needle size ${ }^{c}$} \\
\hline $22 \mathrm{G}$ & 133 & 60 & 45.1 & 22 & 2 & 4 & 1 & 0 & 31 & 0.92 & $(0.74-0.98)$ & 1.00 & $(0.89-1.00)$ \\
\hline $23 \mathrm{G}$ & 173 & 138 & 79.8 & 66 & 7 & 8 & 2 & 0 & 55 & 0.90 & $(0.81-0.95)$ & 1.00 & $(0.93-1.00)$ \\
\hline $25 \mathrm{G}$ & 910 & 664 & 73.0 & 351 & 24 & 39 & 7 & 1 & 242 & 0.94 & $(0.91-0.96)$ & 1.00 & $(0.98-1.00)$ \\
\hline \multicolumn{14}{|l|}{ Necrosis present } \\
\hline Yes & 67 & 41 & 61.2 & 24 & 4 & 9 & 0 & 0 & 4 & 0.86 & $(0.69-0.94)$ & 1.00 & $(0.51-1.00)$ \\
\hline No & 2137 & 1541 & 72.1 & 776 & 65 & 69 & 18 & 5 & 608 & 0.92 & $(0.90-0.94)$ & 0.99 & $(0.98-1.00)$ \\
\hline \multicolumn{14}{|c|}{ Pathologist caseload } \\
\hline$<100$ cases & 164 & 104 & 63.4 & 51 & 7 & 5 & 4 & 0 & 37 & 0.88 & $(0.77-0.94)$ & 1.00 & $(0.91-1.00)$ \\
\hline $100-500$ cases & 651 & 474 & 72.8 & 227 & 29 & 27 & 3 & 2 & 186 & 0.89 & $(0.84-0.92)$ & 0.99 & $(0.96-1.00)$ \\
\hline$>500$ cases & 1389 & 1004 & 72.3 & 522 & 33 & 46 & 11 & 3 & 389 & $0.94^{d}$ & $(0.92-0.96)$ & 0.99 & $(0.08-1.00)$ \\
\hline \multicolumn{14}{|c|}{ First primary Breslow thickness } \\
\hline$\leq 2 \mathrm{~mm}$ & 950 & 705 & 74.2 & 332 & 23 & 34 & 8 & 4 & 304 & 0.94 & $(0.90-0.96)$ & 0.99 & $(0.97-0.99)$ \\
\hline$>2 \mathrm{~mm}$ & 919 & 662 & 72.0 & 367 & 38 & 33 & 6 & 0 & 218 & 0.91 & $(0.87-0.93)$ & 1.00 & $(0.98-1.00)$ \\
\hline Unknown & 335 & 215 & 64.2 & 101 & 8 & 11 & 4 & 1 & 90 & 0.93 & $(0.86-0.96)$ & 0.99 & $(0.94-1.00)$ \\
\hline \multicolumn{14}{|c|}{ First primary ulceration } \\
\hline Yes & 571 & 407 & 71.3 & 247 & 16 & 18 & 1 & 0 & 125 & $0.94^{e}$ & $(0.90-0.96)$ & 1.00 & $(0.97-1.00)$ \\
\hline No & 1009 & 757 & 75.0 & 360 & 40 & 35 & 10 & 4 & 308 & 0.90 & $(0.87-0.93)$ & 0.99 & $(0.97-1.00)$ \\
\hline Unknown & 624 & 418 & 67.0 & 193 & 13 & 25 & 7 & 1 & 179 & 0.94 & $(0.90-0.96)$ & 0.99 & $(0.97-1.00)$ \\
\hline First primary le & n mitot & rate $\left(/ \mathrm{mm}^{2}\right)$ & & & & & & & & & & & \\
\hline$\leq 1$ & 414 & 300 & 72.5 & 136 & 10 & 12 & 4 & 2 & 136 & 0.93 & $(0.88-0.96)$ & 0.99 & $(0.95-1.00)$ \\
\hline 1 to $<4$ & 481 & 352 & 73.2 & 170 & 19 & 20 & 2 & 1 & 140 & 0.90 & $(0.85-0.93)$ & 0.99 & $(0.96-1.00)$ \\
\hline 4 to $<8$ & 332 & 237 & 71.4 & 141 & 7 & 8 & 1 & 0 & 80 & 0.95 & $(0.91-0.98)$ & 1.00 & $(0.95-1.00)$ \\
\hline$\geq 8$ & 392 & 296 & 75.5 & 175 & 22 & 14 & 4 & 0 & 81 & 0.89 & $(0.84-0.93)$ & 1.00 & $(0.95-1.00)$ \\
\hline Unknown & 585 & 397 & 67.9 & 178 & 11 & 24 & 7 & 2 & 175 & 0.94 & $(0.90-0.97)$ & 0.99 & $(0.96-1.00)$ \\
\hline First primary le & n histol & gic subtype & & & & & & & & & & & \\
\hline Desmoplastic & 130 & 97 & 74.6 & 42 & 6 & 6 & 3 & 0 & 40 & 0.88 & $(0.75-0.94)$ & 1.00 & $(0.91-1.00)$ \\
\hline SSM & 512 & 370 & 72.3 & 168 & 10 & 22 & 4 & 1 & 165 & 0.94 & $(0.90-0.97)$ & 0.99 & $(0.97-1.00)$ \\
\hline NM & 461 & 343 & 74.4 & 198 & 20 & 14 & 4 & 1 & 106 & 0.91 & $(0.86-0.94)$ & 0.99 & $(0.95-1.00)$ \\
\hline Other & 754 & 549 & 72.8 & 277 & 26 & 22 & 4 & 1 & 219 & 0.91 & $(0.88-0.94)$ & 1.00 & $(0.97-1.00)$ \\
\hline Unknown & 347 & 223 & 64.3 & 115 & 7 & 14 & 3 & 2 & 82 & 0.94 & $(0.89-0.97)$ & 0.98 & $(0.92-0.99)$ \\
\hline
\end{tabular}


TABLE 6. Continued

\begin{tabular}{|c|c|c|c|c|c|c|c|c|c|c|c|c|c|}
\hline Factor & $\mathrm{N}$ & Confirmed & $\%$ & $\mathrm{TP}$ & $\mathrm{FN}$ & TS & FS & FP & $\mathrm{TN}$ & SN & $(95 \% \mathrm{CI})$ & SP & $(95 \% \mathrm{CI})$ \\
\hline \multicolumn{14}{|c|}{ First primary lesion predominant cell type } \\
\hline Epithelioid & 443 & 329 & 74.3 & 185 & 17 & 13 & 4 & 0 & 110 & 0.92 & $(0.87-0.95)$ & 1.00 & $(0.97-1.00)$ \\
\hline Spindle & 137 & 89 & 65.0 & 47 & 6 & 9 & 0 & 0 & 27 & 0.89 & $(0.77-0.95)$ & 1.00 & $(0.88-1.00)$ \\
\hline Mixed & 173 & 111 & 64.2 & 58 & 6 & 8 & 3 & 0 & 36 & 0.91 & $(0.81-0.96)$ & 1.00 & $(0.90-1.00)$ \\
\hline Unknown & 1451 & 1053 & 72.6 & 510 & 40 & 48 & 11 & 5 & 439 & 0.93 & $(0.90-0.95)$ & 0.99 & $(0.97-1.00)$ \\
\hline
\end{tabular}

TP, true positive; FN, false negative; TS, true suspicious; FN, false negative; FP, false positive; TN, true negative; SN, sensitivity; SP, specificity; 95\% CI, 95\% confidence interval; SSM, superficial spreading melanoma; NM, nodular melanoma.

${ }^{a}$ FNBs that used immunostains had significantly increased sensitivity compared with those that did not $(\mathrm{z}=4.8, \mathrm{P}<.001)$.

${ }^{b}$ FNBs obtained in one pass had significantly increased sensitivity compared with FNBs which required more than one pass $(\mathrm{z}=4.8, \mathrm{P}<$ $.001)$.

${ }^{c}$ The size of the needle used for the FNB procedure was not known in 988 cases.

${ }^{d}$ FNBs reviewed by a cytopathologist who reported $>500$ cases had significantly increased sensitivity compared with FNBs reported by cytopathologists who had reported $<500$ cases $(\mathrm{z}=2.4, \mathrm{P}=.02)$.

${ }^{e}$ FNBs obtained from patients with an ulcerated first primary lesion had increased sensitivity compared with FNBs from patients without ulcerated first primary lesions $(\mathrm{z}=1.9, \mathrm{P}=.05)$.

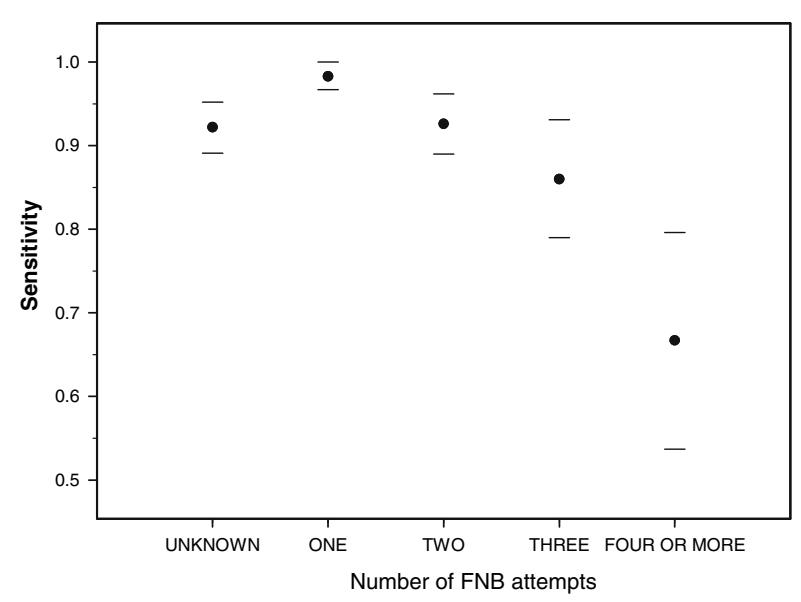

FIG. 3. Sensitivity of fine needle biopsy (FNB) in the diagnosis of metastatic melanoma. Sensitivity is reduced in a linear manner as the number of FNB attempts needed to obtain the sample increases. Bars, $95 \%$ confidence intervals.

of $96.1 \%)$. Other similar case series did not have the power to determine the true diagnostic accuracy of FNB because of their low numbers of FNBs (between 56 and 108 confirmed cases). ${ }^{7-10}$ Voit and colleagues ${ }^{12}$ examined 739 FNBs for metastatic melanoma and found the technique to be highly sensitive (97.9\%) at their institution. However, regional node basins in their patient population were routinely evaluated by ultrasound B-scan, and a third of FNB cases were performed under ultrasound guidance.

Studies of FNB are frequently retrospective investigations and thus verification bias may be a hazard in these studies. Not only are there different reference tests, which are determined by the results of the FNB procedure, namely histopathology after surgical resection for all positive FNB tests and clinical follow-up for most negative procedures, but verification is also partial. Not every consecutive FNB can be verified. Differential verification can lead to overestimation of the measures of diagnostic accuracy. ${ }^{17,18}$ This bias is related to the quality of the reference tests. Confirmation of melanoma metastases by histopathologic examination of tissue removed after surgery is better at identifying true disease status than clinical follow-up of patients with negative FNB results. ${ }^{19}$ Melanoma metastases may regress spontaneously, resulting in failure to identify all false-negative results by clinical follow-up, or they may cause truly positive findings to be incorrectly classified as false positives if the lesion resolves before it is excised or during follow-up. ${ }^{20,21}$

In this study, there were differences in those FNBs that were verified by the reference tests compared with those that were not. Unconfirmed FNBs were more often performed on nonpalpable lesions in visceral sites, and in patients with advanced disease. Verification rates of negative, positive, and suspicious FNBs were $68.2 \%, 73.9 \%$, and $82.1 \%$, respectively.

Despite the high specificity rate, as a result of the large number of confirmed biopsy samples, five falsepositive findings were detected (Table 4). The cytologic features of these cases have been reviewed in more detail elsewhere. ${ }^{22}$ In all cases, misinterpretation of the cellular material had occurred, three of which were found to be adenocarcinoma. Two of these represented metastatic breast adenocarcinoma in axillary nodes, and one was a metastatic papillary adenocarcinoma of renal origin. Two other falsepositive cases were caused by the misinterpretation of large histiocytes or reactive fibroblasts as metastatic melanoma cells.

In 69 FNBs, no metastatic melanoma cells were identified cytologically; however, the presence of 
metastatic melanoma was subsequently identified by histopathologic examination (false negatives) (Fig. 2). Astute clinical and/or radiologic surveillance results in the detection of small suspicious lymph nodes. Difficulties in performing FNBs on such small suspicious lymph nodes contribute to some of the false-negative diagnoses (particulary small, mobile axillary nodes). In fact, approximately a third ( $\mathrm{n}=$ 21) of these biopsy samples (which were reported as "no malignant cells identified") did not contain any cellular material, suggesting that an absence of sampling of the malignant cells was the reason for the false-negative result. Further investigation is usually necessary to identify the cause of the mass lesion. Surgical examination is usually the next step when the index of clinical suspicion is high.

There were several sources of difficulty in performing the FNB procedure for metastatic melanoma. Often it occurred when there was a failure to locate the suspicious lesion because of its small size or its location was not communicated with adequate precision. Failure to identify metastatic melanoma occurred when cellular material showed too few typical morphologic characteristics of metastatic melanoma, or these characteristics were destroyed or masked by necrosis.

The large number of confirmed FNB in this study permitted the analysis of subgroups. Most FNB for metastatic melanoma were performed in lymph nodes as well as skin and subcutaneous tissues. No difference in sensitivity was noted among these tissue groups. However, FNBs of lymph nodes of the axilla were significantly less sensitive compared with those performed at other sites and with FNBs performed in lymph nodes of the groin and neck. A number of factors are likely to contribute to the low sensitivity of FNBs performed in the axilla, including the greater difficulty in gaining access to and locating lymph nodes, particularly those high in the axilla, and the presence of large amounts of fatty tissue in axillary lymph nodes ("horseshoe" nodes).

Fifteen clinicopathologic factors were examined for their effect on the accuracy of FNB for metastatic melanoma, most of which had no effect on the diagnostic accuracy of the test. However, the following four variables did influence the sensitivity of the FNB procedure. (1) Ulceration of the primary lesion led to a small increase in the sensitivity of the test $(\mathrm{P}=0.05)$. (2) Additional needle passes were performed if the initial sampling failed to obtain sufficient cellular material; thus, the number of needle passes is an indicator of the difficulty in obtaining a sample from a specific lesion. FNBs that were performed in only one needle pass were found to have superior sensitivity than those which required additional passes to obtain an adequate sample. Furthermore, the sensitivity of the test seemed to decrease in a linear manner with each subsequent pass. (3) Training and experience have been shown to significantly influence the interpretation of FNB of the breast, ${ }^{23}$ and in this study, we found that the caseload (i.e., level of experience) of the cytopathologist who reviewed the slides influenced the sensitivity of the test. Those cytopathologists who reviewed $>500$ metastatic melanoma FNB samples in the study period performed better. Those cytopathologists who performed $<100$ reviews seemed to do as well as those who had a caseload of 100 to 500 cases. (4) The use of immunostains was associated with improved sensitivity of the test. However, this may reflect the fact that immunochemistry was only performed in those cases in which a sufficiently high cellular yield was obtained, in which case it may have been the high cellular yield that lead to an improvement in sensitivity, rather than the use of immunostains per se. The diverse cytological presentation of metastatic melanoma may also be a factor in the association of the use of immunochemistry with increased sensitivity. ${ }^{24,25}$ Nasiell et al. ${ }^{26}$ found that immunochemical characterization was necessary to conclusively diagnose $>50 \%$ of metastatic melanomas that presented with an equivocal cytological picture. However, immunological characterization cannot be considered definitive when the FNB lacks typical cytologic features expected of metastatic melanoma. For example, one of the false-positive cases exhibited S100 positivity, whereas two of the false-negative FNBs contained melanoma cells that seemed to be S100 negative. This suggests that cytopathologists should be cautious when reporting the results of immunostains on limited samples.

Our study shows that FNB for metastatic melanoma is a procedure with very high specificity and good sensitivity. Several clinicopathologic factors were found to influence the diagnostic accuracy of the test for metastatic melanoma. These included factors relating to the original primary melanoma lesion, location of the sampled lesion, and factors relating to the performance of the test. The SMU employs an ondemand FNB service with assessment of the cytologic material and delivery of a provisional result to the clinician at the time of the patient's visit. This helps guide subsequent diagnostic and therapeutic measures, and it reduces costs (e.g., by decreasing the need for additional patient visits to the clinic). A multidisciplinary approach involving clinicians, 
pathologists and radiologists enables an efficient and cost-effective management strategy in melanoma patients.

\section{ACKNOWLEDGMENTS}

Anna Doubrovsky was supported by the University of Sydney Cancer Research Fund. Rajmohan Murali is supported by the Cancer Institute NSW Clinical Fellowship Program. The invaluable assistance of Ms. Marjorie Colman, the staff of the Department of Cytology and the Sydney Melanoma Unit at the Royal Prince Alfred Hospital are acknowledged. This study was also supported by the University of Sydney Cancer Research Fund, the Cancer Institute NSW, the National Health and Medical Research Council, and the Melanoma Foundation, University of Sydney.

\section{REFERENCES}

1. Geisinger KR, Stanley MW, Raab SS, et al. Modern Cytopathology. Philadelphia: Churchhill Livingston, 2004.

2. Orell SR, Stenett GF, Walters MN, et al. Manual and Atlas of Fine Needle Aspiration Cytology. Philadelphia: Churchhill Livingston, 1999.

3. Steel BL, Schwartz MR, Ramzy I. Fine needle aspiration biopsy in the diagnosis of lymphadenopathy in 1,103 patients. Role, limitations and analysis of diagnostic pitfalls. Acta Cytol 1995; 39:76-81.

4. Lussier C, Klijanienko J, Vielh P. Fine-needle aspiration of metastatic nonlymphomatous tumors to the major salivary glands: a clinicopathologic study of 40 cases cytologically diagnosed and histologically correlated. Cancer 2000; 90:350-6.

5. Bofin AM, Lydersen S, Isaksen C, Hagmar BM. Interpretation of fine needle aspiration cytology of the breast: a comparison of cytological, frozen section, and final histological diagnoses. Cytopathology 2004; 15:297-304.

6. Logrono R, Kurtycz DF, Sproat IA, Shalkham JE, Stewart JA, Inhorn SL. Multidisciplinary approach to deep-seated lesions requiring radiologically-guided fine-needle aspiration. Diagn Cytopathol 1998; 18:338-42.

7. Basler GC, Fader DI, Yahanda A, Sondak VK, Johnson TM. The utility of fine needle aspiration in the diagnosis of melanoma metastatic to lymph nodes. J Am Acad Dermatol 1997; 36:403-8.

8. Cangiarella J, Symmans WF, Shapiro RL, et al. Aspiration biopsy and the clinical management of patients with malignant melanoma and palpable regional lymph nodes. Cancer 2000; 90:162-6.
9. Rodrigues LK, Leong SP, Ljung BM, et al. Fine needle aspiration in the diagnosis of metastatic melanoma. $J$ Am Acad Dermatol 2000; 42:735-40.

10. Hafstrom L, Hugander A, Jonsson PE, Lindberg LG. Fineneedle-aspiration cytodiagnosis of recurrent malignant melanoma. J Surg Oncol 1980; 15:229-34.

11. Perry MD, Seigler HF, Johnston WW. Diagnosis of metastatic malignant melanoma by fine needle aspiration biopsy: a clinical and pathologic correlation of 298 cases. J Natl Cancer Inst 1986; 77:1013-21.

12. Voit C, Mayer T, Proebstle TM, et al. Ultrasound-guided fineneedle aspiration cytology in the early detection of melanoma metastases. Cancer 2000; 90:186-93.

13. Balch CM, Buzaid AC, Soong SJ, et al. Final version of the American Joint Committee on Cancer staging system for cutaneous melanoma. J Clin Oncol 2001; 19:3635-48.

14. Einstein AJ, Bodian CA, Gil J. The relationships among performance measures in the selection of diagnostic tests. Arch Pathol Lab Med 1997; 121:110-7.

15. Habbema JDF, Eijkemans R, Krijnen P, Knottnerus JA. Analysis of data on the accuracy of diagnostic tests. In: Knottnerus JA. ed. The Evidence Base of Clinical Diagnosis. BMJ Publishing Group, London, 2002: 117-43.

16. Raab SS, Bottles K, Cohen MB. Technology assessment in anatomic pathology. An illustration of test evaluation using fine-needle aspiration biopsy. Arch Pathol Lab Med 1994; 118:1173-80.

17. Lijmer JG, Mol BW, Heisterkamp S, et al. Empirical evidence of design-related bias in studies of diagnostic tests. JAMA 1999; 282:1061-6.

18. Whiting P, Rutjes AW, Reitsma JB, Glas AS, Bossuyt PM, Kleijnen J. Sources of variation and bias in studies of diagnostic accuracy: a systematic review. Ann Intern Med 2004; 140:189-202.

19. Panzer RJ, Suchman AL, Griner PF. Workup bias in prediction research. Med Decis Making 1987; 7:115-9.

20. Katz KA, Jonasch E, Hodi FS, et al. Melanoma of unknown primary: experience at Massachusetts General Hospital and Dana-Farber Cancer Institute. Melanoma Res 2005; $15: 77-82$.

21. Vijuk G, Coates AS. Survival of patients with visceral metastatic melanoma from an occult primary lesion: a retrospective matched cohort study. Ann Oncol 1998; 9:419-22.

22. Murali R, Doubrovsky A, Watson GF, et al. Diagnosis of metastatic melanoma by fine needle biopsy: analysis of 2204 cases. Am J Clin Pathol 2007; 127:385-97.

23. Cohen MB, Rodgers RP, Hales MS, et al. Influence of training and experience in fine-needle aspiration biopsy of breast. Receiver operating characteristics curve analysis. Arch Pathol Lab Med 1987; 111:518-20.

24. Perry MD, Gore M, Seigler HF, Johnston WW. Fine needle aspiration cytology of metastatic melanoma: a morphological analysis of 174 cases. Acta Cytol 1985; 30:386-96.

25. Layfield LJ, Ostrzega N. Fine needle aspirate smear morphology in metastatic melanoma. Acta Cytol 1989; 33:606-12.

26. Nasiell K, Tani E, Skoog L. Fine needle aspiration cytology and immunocytochemistry of metastatic melanoma. Cytopathology 1991; 2:137-47. 